\title{
Variance-Aware Optimal Power Flow
}

\author{
Daniel Bienstock and Apurv Shukla \\ Columbia University, NY, United States \\ \{dano, apurv.shukla\}@ columbia.edu
}

\begin{abstract}
The incorporation of stochastic loads and generation into the operation of power grids gives rise to an exposure to stochastic risk. This risk has been addressed in prior work through a variety of mechanisms, such as scenario generation or chance constraints, that can be incorporated into OPF computations. Nevertheless, numerical experiments reveal that the resulting operational decisions can produce power flows with very high variance. In this paper we introduce a variety of convex variants of OPF that explicitly address the interplay of (power flow) variance with cost minimization, and present numerical experiments that highlight our contributions.
\end{abstract}

Index Terms-Chance-constraints, stochastic generation, OPF.

\section{INTRODUCTION}

Increasing levels of renewable penetration have resulted in associated risk, principally through potential equipment overloads. A growing body of research has tackled this issue through proposed modifications to power engineering practices, in particular by suggesting alternative, risk-aware formulations to the Optimal Power Flow (OPF) problem that may additionally incorporate appropriate balancing mechanisms. See e.g. [1]-[11], [4] and citations therein.

The application of these methodologies yields operational plans within the scope of an OPF computation that guarantee protection from stochastic variations in generation (or loads). For example, in the chance-constrained case, one obtains a set of dispatch decisions and possibly participation factors (which describe balancing control) that guarantee that the probability that any given line becomes overloaded is at most a given value $\epsilon>0$, under appropriate assumptions on renewable stochasticity.

However, experiments with such schemes produce cases where power flows are highly variable, or more precisely some transmission lines will have power flows with high variance. We posit that this behavior is undesirable from a real-time operational perspective. See [12], which highlights the impact of power flow variability on voltage profile and transformer operation. High variability may also hinder system understanding and control, as well as pricing mechanisms (see [13], [14], [15] for work on pricing under uncertainty). [16] considers minimization of variance of slack bus injection.

In this paper we propose a set different alternatives to risk-aware OPF computations, which we term variance-aware $O P F$. In these variants we explicitly account for power flow variance, either as a post-processing step for the initial riskaware OPF computation, or by explicitly incorporating a mea- sure of power flow variance into a risk-aware OPF formulation so as to obtain a convex optimization problem.

\section{NOTATION AND BASIC FORMULATIONS}

In this paper we will focus on the linearized, or DC model for power flows. We will use the following selected nomenclature, with additional terms described later:

$\mathcal{B}=$ set of buses, $n=|\mathcal{B}| ; B=$ bus susceptance matrix.

$\hat{B}=B$, with the last row and column removed

$\breve{B}=\left[\begin{array}{cc}\hat{B}^{-1} & 0 \\ 0 & 0\end{array}\right] ; \breve{B}_{i}=i^{\text {th }}$ row of $\breve{B}$.

$\theta_{i}=$ phase angle at bus $i, \bar{\theta}_{i}=\mathbf{E}\left(\theta_{i}\right)$.

$\mathcal{E}=$ set of lines; $m=|\mathcal{E}|$.

$f_{i j}^{\max }, b_{i j}=$ power flow limit, and susceptance, for line $i j \in \mathcal{E}$.

$f_{i j}=$ power flow on line $i j, \bar{f}_{i j}=\mathbf{E}\left(f_{i j}\right)=b_{i j}\left(\bar{\theta}_{i}-\bar{\theta}_{j}\right)$

$\pi_{i j} \doteq \breve{B}_{i}^{T}-\breve{B}_{j}^{T}$ for each line $i j \in \mathcal{E}$

$\mathcal{G}=$ set of generator buses; we assume at most one generator per bus

$p_{i}^{\min }, p_{i}^{\max }=$ minimum and maximum output of $i \in \mathcal{G}$.

$\mathcal{S}=$ set of stochastic injection buses, $\mu_{k}+\omega_{k}=$ stochastic injection at bus $b \in \mathcal{B}$,

- $\mu_{k}=$ constant, $\omega_{k}=$ zero-mean random variable.

- $\mu_{k}=\omega_{k}=0$ if $k \notin S$,

- $\Omega=$ covariance of $\omega$, an $n \times n$ matrix,

- $\mathcal{W}=$ set of vectors $\omega$ under consideration.

$\mathcal{R}=$ set of buses participating in balancing

$\mathcal{A}=$ matrix of participation factors; $\alpha_{i j}=(\mathrm{i}, \mathrm{j})$-entry of $\mathcal{A}$,

- $\mathcal{A}$ is $n \times n$

- $\alpha_{i j}=0$ if $i \notin \mathcal{R}$ or $j \notin \mathcal{S}$

- $\mathbf{V}(\mathcal{A})=$ vector with entries $b_{i j}^{2} \pi_{i j}^{T}(I-\mathcal{A}) \Omega\left(I-\mathcal{A}^{T}\right) \pi_{i j}$ (variances) for $i j \in \mathcal{E}$

$\mathcal{K}=$ convex set of allowable participation factor matrices $p=$ vector of generation amounts, extended to all $b \in \mathcal{B}$

(forcing $p_{b}=0$ when $b \notin \mathcal{B}$ ).

$c(p)=($ convex $)$ cost of generation vector $p$.

$d=$ (fixed) vector of loads.

Using this notation, we can write a DC-OPF formulation:

$$
\begin{array}{ll} 
& \min _{p} c(p) \\
\text { s.t. } & B \theta=p-d \\
& \forall i j \in \mathcal{E}: \quad b_{i j}\left|\theta_{i}-\theta_{j}\right| \leq f_{i j}^{\max } \\
& \forall i \in \mathcal{G}: p_{i}^{\text {min }} \leq p_{i} \leq p_{i}^{\text {max }}
\end{array}
$$

As is well-known, constraints (1b)-(1c) can be simplified by using a pseudo-inverse for the matrix $B$ (see, e.g. [1], [3]). 
Namely, for each line $i j$ the power flow on $i j$ equals $b_{i j} \pi_{i j}^{T}(p-$ $d)$ thereby reducing (1b)-(1c) to the system

$$
\forall i j \in \mathcal{E}: b_{i j}\left|\pi_{i j}^{T}(p-d)\right| \leq f_{i j}^{\max }
$$

\section{SECURITY-CONSTRAINED FORMULATIONS}

Previous work [1], [3], [4], [9], [17], [7], [2], [5] has focused on modifications to power flow computations in order to account for uncertain injections, and in particular to model the use of balancing. We will first outline some of this work.

Second, we will also describe a sparse formulation for security-constrained DC-OPF problems, as a straightforward extension of the approach used in [1], [3], [9]. This will be the starting point for the discussions in Section $\mathrm{V}$, where we will discuss how to make the formulation variance-aware.

The method used in [1], [3], [9], modifies the DC-OPF computation so that its primary output consists of a vector $\bar{p}$ of controllable generation amounts (which can only be positive at controllable generators) and (as in [9]) an $n \times n$ matrix $\mathcal{A}$ used to model balancing. Given $\omega \in \mathcal{W}$ the balancing vector is given by $\mathcal{A} \omega$, so that the stochastic output of a controllable generator at bus $i$ is

$$
p_{i}=p_{i}(\omega)=\bar{p}_{i}-[\mathcal{A} \omega]_{i}=\bar{p}_{i}-\sum_{j \in \mathcal{B}} \alpha_{i j} \omega_{j}
$$

where we assume that when $i$ is not a controllable generator bus then $\alpha_{i j}=0$ for all $j$. Thus, the net injection vector equals $\bar{p}-d+\mu+\omega-\mathcal{A} \omega$. In order to actually attain balancing we must have

$$
\forall w \in \mathcal{W}: \quad \sum_{i \in \mathcal{B}}(\bar{p}-d+\mu+\omega-\mathcal{A} \omega)_{i}=0,
$$

a stochastic requirement. Let us assume that we additionally assume $\sum_{i \in \mathcal{B}}(\bar{p}-d+\mu)_{i}=0$, i.e. the system is balanced when $\omega=0$. Then (4) is equivalent to

$$
\forall \omega \in \mathcal{W}: \quad \omega_{i}-\sum_{j \in \mathcal{B}} \alpha_{i j} \omega_{j}=0, \quad \forall i \in \mathcal{B} .
$$

As pointed out in [9] in order to attain this condition it is sufficient to require

$$
1=\sum_{j \in \mathcal{B}} \alpha_{i j} \quad \forall i \in \mathcal{B} .
$$

Note that (5) describes a hyperplane in $\omega$-space. If $\mathcal{W}$ is full dimensional then (6) is actually necessary for (4) [18]. Additionally we may constrain $\mathcal{A}$ in a number of ways (e.g. the $\alpha_{i j}$ constrained by bounds). In particular, we could require that for any bus $i \in \mathcal{R}{ }^{1} \alpha_{i j}=\alpha_{i k}$ for every $j \neq k$ (termed a "global" policy in [9]).

Notation. In what follows we will write $\mathcal{A} \in \mathcal{K}$ to denote a set of generic convex constraints that $\mathcal{A}$ must satisfy, including in particular (6).

\footnotetext{
${ }^{1}$ Henceforth, a participating bus.
}

Using these notations, if $f_{i j}$ denotes (stochastic) flow on line $i j$ then

$$
\begin{array}{ll}
\forall i j \in \mathcal{E}: \quad & f_{i j}=b_{i j} \pi_{i j}^{T}(\bar{p}-d+\mu+\omega-\mathcal{A} \omega), \\
& \mathbf{E}\left(f_{i j}\right)=b_{i j} \pi_{i j}^{T}(\bar{p}-d+\mu) .
\end{array}
$$

Likewise by construction

$$
\begin{aligned}
& \mathbf{V}(\mathcal{A})_{i j} \doteq \operatorname{Var}\left(f_{i j}\right)=b_{i j}^{2} \operatorname{Var}\left(\pi_{i j}^{T}(I-\mathcal{A}) \omega\right)= \\
& \quad b_{i j}^{2} \pi_{i j}^{T}(I-\mathcal{A}) \Omega\left(I-\mathcal{A}^{T}\right) \pi_{i j}
\end{aligned}
$$

The next modeling ingredient concerns security constraints. A variety of variants of the basic problem (1) can be obtained depending on how we model stochasticity and security and on whether the matrix $\mathcal{A}$ is an optimization variable or is fixed (i.e. given as an input). A typical approach concerns chance constraints. To fix ideas, consider a line $i j$. Then we wish to impose that

$$
\mathbf{P}\left(\left|f_{i j}\right|>f_{i j}^{\max }\right)<\epsilon
$$

where $0<\epsilon<1$ is a given threshold. In order to represent (9) in a convex manner, prior work has assumed normally distributed of $\omega$. Under such an assumption (9) is equivalent to

$$
\left|\mathbf{E}\left(f_{i j}\right)\right|+\Phi^{-1}(1-\epsilon) \mathbf{S t d}\left(f_{i j}\right) \leq f_{i j}^{\max }
$$

where $\Phi^{-1}(1-\epsilon)$ is the $\epsilon$-quantile for a normal distribution. Using (7) and (8), we obtain that (10) is SOCP representable [1], [3] (also see below).

\section{A. Modifications used in this paper}

Our first modification will be to replace (10) with

$$
\left|\mathbf{E}\left(f_{i j}\right)\right|+\nu_{i j} \mathbf{S t d}\left(f_{i j}\right) \leq f_{i j}^{\max }
$$

where $\nu_{i j}$ is a safety parameter. As seen above, in the Gaussian case (11) is equivalent to 97 if we choose $\nu_{i j}=\Phi^{-1}(1-\epsilon)$. However, the Gaussian case is not the only one where such an equivalence holds; other examples of distributions of interest include (multivariate) truncated Gaussians, uniform distributions on ellipsoidal supports, and others. This requires a distribution-dependent choice $\nu_{i j}=\nu_{i j}(\epsilon)$. Further (11) can be used to tightly approximate distributionally robust chance constraints. See [19] (and e.g. Theorem 2.1 therein). Similar remarks apply to security constraints involving generators. We would argue that even when the stochastics of $\omega$ is complex so that we cannot provide a rigorous choice for the safety parameters, we may still be able to compute $\Omega$, or perhaps a data-driven estimate for it. The safety-parameter approach could still hold appeal from an intuitive, if imprecise, perspective. We will term (11) a safety constraint.

In order to present our modification to chance-constrained DC-OPF, we first produce a new expression for the variance of a line flow $f_{i j}$. Let us write

$$
\begin{aligned}
D & \doteq \breve{B} \mathcal{A}, \quad \text { and } \\
\forall k \in \mathcal{S}: \gamma_{i j, k} & \doteq \breve{B}_{i k}-\breve{B}_{j k}-D_{i k}+D_{j k} .
\end{aligned}
$$


Lemma 1. For any line $i j$, the variance of flow on ij under scheme (3) is given by

$$
\operatorname{Var}\left(f_{i j}\right)=b_{i j}^{2} \gamma_{i j} \Omega \gamma_{i j}^{T}
$$

Remark: we stress that this expression holds without any assumption on the underlying probability distributions. Denote the generation cost at a bus $i$ as $c_{i}(p) \doteq c_{i 0} p^{2}+c_{i 1} p+c_{i 0}$, and let the $i^{\text {th }}$ row of $\mathcal{A}$ be denoted by $\mathcal{A}_{i}$. Then (routine proof):

Lemma 2. For a bus $i, \operatorname{Var}\left(p_{i}\right)=\mathcal{A}_{i}^{T} \Omega \mathcal{A}_{i}$, and $\mathbf{E}\left(c_{i}\left(p_{i}\right)\right)=$ $c_{i 0}\left(\bar{p}_{i}^{2}+\mathcal{A}_{i}^{T} \Omega \mathcal{A}_{i}\right)+c_{i 1} \bar{p}_{i}+c_{i 2}$.

Our safety-constrained formulation, given next, generalizes the chance-constrained formulation in [3]. As before we write $n=|\mathcal{B}|$ and also $m=|\mathcal{E}|$. We are given nonnegative a safety parameter $\nu_{i j}$ for each line $i j$ and likewise safety parameters $\nu_{i}$ for generators ( $=0$ at non-generator buses). The formulation uses variables $\bar{p}, \bar{\theta}$ ( $n$-vectors), $\bar{f}$ (m-vector), $\mathcal{A}, D(|\mathcal{S}| \times|\mathcal{S}|$ and $n \times|\mathcal{S}|$ matrices, respectively), and $\gamma$ and $s$ (an $m \times|\mathcal{S}|$ matrix and $m$-vector, respectively). As above, we use $\mathcal{A} \in \mathcal{K}$ to denote a given set of convex constraints on $\mathcal{A}$.

$$
\begin{aligned}
& \min \sum_{i \in \mathcal{G}} \mathbf{E}\left(c_{i}\left(p_{i}\right)\right) \\
& \text { s.t. } \mathcal{A} \in \mathcal{K} \\
& B \bar{\theta}=\bar{p}+\mu-d \\
& \bar{f}_{i j}=b_{i j}\left(\theta_{i}-\bar{\theta}_{j}\right) \\
& b_{i j}\left|\bar{f}_{i j}\right|+\nu_{i j} s_{i j} \leq f_{i j}^{\max } \quad \forall i j \in \mathcal{E}, \\
& \breve{B} \mathcal{A}=D \\
& \gamma_{i j, k}=\breve{B}_{i, k}-\breve{B}_{j, k}-D_{i, k}+D_{j, k}, \quad \forall i j \in \mathcal{E}, k \in \mathcal{B} \\
& s_{i j} \geq b_{i j} \sqrt{\gamma_{i j} \Omega \gamma_{i j}^{T}} \quad \forall i j \in \mathcal{E} \\
& \forall i \in \mathcal{G}: \\
& p_{i}^{\text {min }}+\nu_{i} \sqrt{\mathcal{A}_{i}^{T} \Omega \mathcal{A}_{i}} \leq \bar{p}_{i} \leq p_{i}^{\max }-\nu_{i} \sqrt{\mathcal{A}_{i}^{T} \Omega \mathcal{A}_{i}} .
\end{aligned}
$$

Equations (7) and Lemma (1), together with constraints (15c)(15h) imply that $(15 \mathrm{e})$ correctly states the desired line safety constraint. Similarly with the generator safety constraints. Problem (15) is a convex quadratically constrained optimization problem that can, in principle, be solved using standard optimization software. We call this the sparse formulation. Next we analyze the structure of this formulation, in light of the fact that previous work [9], [3], [17] has highlighted numerical difficulties in solving chance-constrained formulations for relatively large systems. Thus it is important to point out that our formulation in fact can be greatly reduced in size.

In particular, as denoted in Section II and in the remarks following Lemma 1 if a bus $j \notin S$ then $\alpha_{i j}=0$ for every bus $i$, and if bus $i \notin \mathcal{R}$ then $\alpha_{i j}=0$ for every $j$. In summary we have:

Lemma 3. In the sparse formulation, the number of $\mathcal{A}, D$ and $\gamma$ variables is, respectively, $|\mathcal{R}||\mathcal{S}|, n|\mathcal{S}|$ and $m \mathcal{S} \mid$. In addition we also have the $\bar{\theta}, \bar{f}$ and $p$ variables, which number $2 n+m$.
The number of nonzeros in all constraints (15f) and 15h is $n|\mathcal{R}||\mathcal{S}|$ and $m|\mathcal{S}|$, respectively.

We note here are several variants of the above safetyconstrained problem that could be meaningful. First, there has been recent work on how to avoid the normality assumption [20]. Another alternative would be to rely on scenario modeling (see e.g. [2]), or to use a distributionally robust model with underlying normality [4].

\section{A NUMERICAL EXAMPLE}

In this section we consider some simple examples of security-constrained problems 15 so as to examine the tradeoff between cost and various measures of line flow variance. Consider the following example:

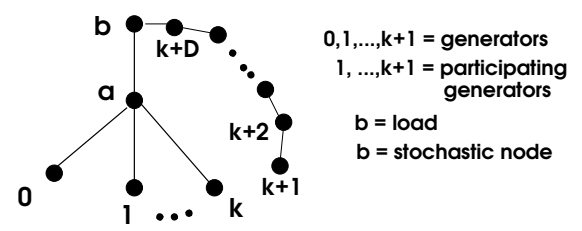

Figure 1. High-variance example.

Here,

- Quantities $k$ and $D$ are large

- Bus $b$ has a load of $L$ units.

- The stochastic output at bus $b$ is indicated by $\omega$, with mean $\mu<L$ and variance $\sigma^{2}$.

- Bus $i(0 \leq i \leq k+1)$ is a generator with linear cost function given by $c_{i 1} p_{i}$.

Assume $c_{01}<c_{11}=c_{21} \ldots c_{k 1}<c_{k+1,1}$.

- The generator at bus 0 is large (capacity larger than $L$ ) and non-participating.

- Generators at buses $1,2, \ldots, k+1$ are all participating, with zero lower limit and safety parameters of value 3 .

- Buses $a, k+1, \ldots, k+D$ have no load, no generation and no stochastic injection.

- We assume (for the first analysis) that all line limits are large.

Let us write the participating factor for generator $i \geq 1$ as $\alpha_{i}$. Consider the following candidate solution:

$$
\begin{aligned}
& \bar{p}_{0}=L-\mu-3 \sigma \\
& \alpha_{i}=1 / k \text { and } \bar{p}_{i}=3 \sigma / k, \text { for } 1 \leq i \leq k . \\
& \alpha_{k+1}=\bar{p}_{k+1}=0 .
\end{aligned}
$$

Lemma 4. The solution stipulated by (16) is the unique optimal solution for the case of problem (15) in Figure 1

Proof. First we argue that the solution we gave is feasible. The generation at bus $i(1 \leq i \leq k)$ equals $3 \sigma / k-\omega / k$. Hence the security constraint at bus $i$ is satisfied. Also, total generation equals $L-3 \sigma+3 \sigma-\omega=L$. Thus indeed the solution is feasible. Now consider any other feasible solution given by values $\hat{p}_{i}$ and $\alpha_{i}$ (average generation and participating factor 
at bus $i$ ). Define $P=\sum_{i=1}^{k+1} \hat{p}_{i}$. Then $\hat{p}_{0}=L-\mu-P$, and so the cost of the solution is

$$
\begin{aligned}
& c_{01}(L-\mu-P)+c_{11}\left(P-p_{k+1}\right)+c_{k+1,1} \hat{p}_{k+1} \geq \\
& c_{01}(L-\mu-P)+c_{11} P .
\end{aligned}
$$

Here the inequality is strict unless $p_{k+1}=0$. Further, $\hat{p}_{i} \geq$ $3 \alpha_{i} \sigma$ and $\sum_{i} \alpha_{i}=1$. So, $P \geq 3 \sigma$. The proof is now complete.

Let us now consider the stochastic flow on line $a b$, which equals $L-\mu-\omega$, and therefore has variance $\sigma^{2}$. In other words line $a b$ is exposed to $100 \%$ of stochastic injection variance. (Additional comments, below). The sum of line flow variances equals $\sigma^{2}(1+1 / k) \approx \sigma^{2}$ for $k$ large.

Suppose we were to aim for a decrease of variance on this line by $50 \%$. This goal will be achieved by setting $\sum_{i=1}^{k} \alpha_{i}=\sqrt{.5}$ and thus $\alpha_{k+1} \approx 0.293$. In that case the sum of variances will be larger than $.5 \sigma^{2}+(D+1) \alpha_{k+1}^{2} \sigma^{2} \approx\left(.5+(.293)^{2}(D+1)\right) \sigma^{2}$. With $D=10$ this quantity equals approximately $1.44 \sigma^{2}$ and the sum of line flow variances has substantially increased!

In the previous example we assumed that line limits were large. However, it is simple to adapt the example to that were line limits are small enough that the safety constraints become significant. At the same time, it is of interest to consider an alternative variance metric that takes into account line limits. A compelling metric is the sum, over all lines, of the ratio of flow variance to square of line limit. To address this metric we modify the data in the example above by assuming:

- $\mu=L / 4$ and $\sigma=\mu / 2=L / 8$.

- The limit for lines $0 a$ and $a b$ equals $9 L / 8$.

- The limit for all lines $i a(1 \leq i \leq k)$ and for the lines on the path from $k+1$ to $b$ are all equal to $2 \sigma$.

- All line safety parameters have value 3 .

We can verify that the solution given by 16 remains feasible, in which case a close reading of Lemma 4 shows that it remains the sole optimal solution. To verify feasibility, note that the expected flow on line $a b$ equals $3 L / 4$ and its standard deviation equals $L / 8$; since the limit for that line is $9 L / 8$ it follows that the safety constraint is satisfied (exactly), and likewise with the remaining lines. Thus feasibility of 16 holds, and it is straightforward to show that Lemma 4 still holds.

Further, let us consider the variance metric (sum of ratios of line flow variances to square of line limits) in solution (16). Line $a b$ contributes $1 / 81$ to the sum whereas each line ia contributes equals $1 /\left(4 k^{2}\right)$. Hence the total metric equals $1 / 81+1 /(4 k) \approx .0123$ for $k$ large. If, as above we desire to decrease the variance on line $a b$ by $50 \%$ then we again set $\alpha_{k+1}=1-\sqrt{.5} \approx .293$. We also set $\bar{p}_{k+1}=3 \alpha_{k+1}$. Since the line limit on the path from $k+1$ to $b$ is $2 \sigma>6 \alpha_{k+1}$ it follows that this solution is indeed feasible for (15). The variance metric for the new solution is (more than) $0.5 / 81+(.293)^{2}(D+1) / 4 \approx 0.242$. In other words, in our attempt to reduce variance in one line we have worsened the variance metric by approximately a factor of 20 . If we reduce $D$ from 10 to 3 the metric for the new solution is approximately 0.098 and thus more than 8 times larger than that for the original solution.

To conclude this section we point out that:

1) In either case, a cursory analysis might seem to reveal that any solution to the security-constrained problem will concentrate variance on line $a b$. That is not so: as we have shown, we can shift variance to the path from bus $k+1$ to $b$. This approach, however, both increases cost and system variance. Also note that on the surface the network design above makes sense: cheaper participating generators are closer to the load.

2) The system we have discussed has been expressly designed so as to evince an extreme behavior. And, of course, different parameter choices will result in less extreme behavior. However we caution the reader that the example can be modified by introducing more participating generators and additional loads, with the appropriate cost and security structure, to obtain a system setup that is not at first glance extreme, while the actual behavior does become even more extreme than the one we provided. Moreover the qualitative behavior we have discussed can be seen in realistic examples.

3) The behavior we see in the example, in summary, is one where network topology, load structure (i.e. location of loads, stochastic injection nodes and responding buses) and cost structure conspire so as to concentrate variance on a specific line or more generally a small set of lines. As the example shows, we can expect an inherent set of tradeoffs between operational cost, system-wide variance metrics, and variance on selected ("important") lines. This tradeoff is one that should be visible to and controllable by system operators. Next section addresses this point.

\section{VARIANCE-AWARE PROBLEMS}

In this section we consider modifications to the process of solving formulation (15), so as to capture tradeoffs with variance metrics. As a starting point in this direction, we formulate an optimization problem of the general type

$$
\begin{aligned}
& \min \sum_{i \in \mathcal{G}} \mathbf{E}\left(c_{i}\left(p_{i}\right)\right)+\Delta\left(\bar{f}, s^{2}\right) \\
& \text { s.t. } 15 \mathrm{~b}-15 \mathrm{i}
\end{aligned}
$$

where $s^{2}$ is the vector with entries $s_{i j}^{2}$ (variances of line flows), and

$$
\Delta\left(\bar{f}, s^{2}\right)=\sum_{i j \in \mathcal{F}} \Delta_{i j}\left(\bar{f}_{i j}, s_{i j}^{2}\right),
$$

Here, $\mathcal{F} \subseteq \mathcal{E}$ and each $\Delta_{i j}$ is a nonnegative function chosen to highlight a specific penalty as a function of expected flow and variance. In other words, we impose all constraints of the safety-constrained problem (15) but we add to the objective a term that enforces a tradeoff with variance. The function $\Delta$ will be our formal "variance metric." 


\section{A. Some variance metrics}

Next we describe some concrete models of variance metrics (20).

(I) $\mathcal{F}=\mathcal{E}$, and for all $i j \in \mathcal{E}, \Delta_{i j}$ is convex and nondecreasing in $s_{i j}^{2}$. As a special case, let $\Delta_{i j}\left(\bar{f}_{i j}, s_{i j}\right)=\psi_{i j} s_{i j}^{2}$ where $\psi_{i j} \geq 0$. When $\psi_{i j}=1$ or $\psi_{i j}=\left(1 / f_{i j}^{\max }\right)^{2}$ $\Delta\left(\bar{f}, s^{2}\right)$ is the metric we considered in the examples given in Section IV.

(II) More complex models are those where $\mathcal{F}=\mathcal{F}\left(\bar{f}, s^{2}\right)$. For example let $N>0$ be given, and consider a function $\Delta$ of the form

$$
\Delta(\bar{f}, s)=\sum_{i j \in \mathcal{F}} \psi_{i j} s_{i j}^{2}
$$

where again the $\psi_{i j} \geq 0$ are scalars, and $\mathcal{F} \subseteq \mathcal{E}$ is a set of lines, such as

(II.1) The set of $N$ lines with largest flow magnitude.

(II.2) The set of $N$ lines with largest flow variance. In either model, we do not know in advance the set $\mathcal{F}$ to be summed over, yet the objective is relevant.

(III) A very different class of convex models is that where for each $i j$ we define $\Delta_{i j}\left(\bar{f}_{i j}, s_{i j}\right)$ as follows:

$$
\begin{aligned}
\Delta_{i j} & =-\rho_{i j} \log \left(s_{i j}^{2}-b_{i j}^{2} \gamma_{i j} \Omega \gamma_{i j}^{T}\right), \quad \text { if } s_{i j}^{2}>b_{i j}^{2} \gamma_{i j} \Omega \gamma_{i j}^{T} \\
& =+\infty, \quad \text { otherwise. }
\end{aligned}
$$

where $\rho_{i j}>0$ is a parameter. This is the classical logarithmic barrier formulation [21]. Because of the definition of the $\Delta_{i j}$, the conic constraints $(15 \mathrm{~h}$ can be removed. It is known that if the $\rho_{i j}$ are all equal to a common value $\rho$, then the solution to (19) (with (15h) removed) converges to an optimal solution to the security-constrained problem (15) as $\rho \rightarrow 0^{+}$. For a fixed choice of the $\rho_{i j}$ we obtain a ranking of the importance of (security of) individual lines, plus a tradeoff against generation cost. The formulation (19) may thus be seen as a viable alternative to safety-constrained formulations, without the computational burden of the conic constraints (15h).

In model (I) we obtain a standard second-order cone program. Model (III) is also convex, though nonstandard (i.e. not an SOCP). Model (II.2) can be formulated as a convex program (proof omitted).

\section{B. A correction template}

Our goal in this work is to address the tradeoff between generation cost, security constraints and variance metrics, and one way to do so is to solve the optimization problem (19), which is in principle a straightforward task in model (I). However, previous experience with chance-constrained DCOPF [3], [6] indicates that a direct solution approach relying on state-of-the-art solvers is likely to fail due to numerical difficulties. Our numerical tests with formulation (19) in model (I) verifies this fact.

As an alternative to formal optimization we instead focus on a procedure that seeks to correct or adjust the solution to the non-variance aware safety-constrained problem (15). A template for the overall scheme is as follows.

\section{Template V.1. GENERIC CORRECTION TEMPLATE}

Input: an instance of the safety-constrained problem (15) and a variance metric.

Step I. Solve (15), with solution $\left(\bar{p}^{*}, \mathcal{A}^{*}\right)$.

Step II. Perform a small number of adjustment iterations which shift $\left(\bar{p}^{*}, \mathcal{A}^{*}\right)$ to a new feasible solution to (15) that attains an improved value of the variance metric, while at the same time increasing generation cost in a moderate manner.

We will describe an implementation of Step II where we perform first- or second-order steps that amount to solving convex optimization problems which do not include large numbers of conic constraints.

Prior to describing the specific implementation we briefly discuss the motivation for using this template. As has been observed in prior work, in the solution to typical chanceconstrained problems similar to (15), only very a small number of the conic constraints $15 \mathrm{~h}$ are binding or nearly binding, that is to say, only a small number of safety constraints are active. If some of those lines are among those with largest flow (literally, a handful) we expect that their variance will also be fairly small. However, there generally is a relatively large number of lines with quite large variance of flow. It follows that there is "low hanging fruit", i.e. opportunities for shifting variance so as to improve a given variance metric. In doing so, we may slightly increase the variance of lines with nearly-binding safety-constraints. This increase may require a correspondingly slight decrease of flow in such lines, so as to satisfy the safety constraints. Flow will thus be shifted onto other lines, possibly resulting in a small increase in cost. These expectations are borne out in experiments detailed below, and they form the underpinning for the above template.

\section{A specific implementation for the template}

Here we describe an implementation of the above template. For simplicity of exposition, we assume that

(1) For each line $i j, \Delta_{i j}\left(\bar{f}_{i j}, s_{i j}^{2}\right)=\Delta_{i j}\left(s_{i j}^{2}\right)$, i.e. it is a function of line variance only. Further, it is assumed that $\Delta_{i j}\left(s_{i j}^{2}\right)$ is convex and nondecreasing.

(2) The set $\mathcal{F}$ used to define $\Delta\left(\bar{f}, s^{2}\right)$ in 20 does not depend on the variances, i.e. $\mathcal{F}=\mathcal{F}(\bar{f})$.

Assumptions (1) and (2) match models (I) and (II.1) above. However we stress that there are variants of the procedures below that handle cases (II.2) and (III).

Definition V.2. Let $\bar{f}$ be a power flow vector and let $\mathcal{A} \in K$ be a matrix of participation factors. We will say that the pair $(\bar{f}, \mathcal{A})$ is compatible (or that $\mathcal{A}$ is compatible with $\bar{f}$ ) 
for problem (15), if there exist $\bar{p}, \bar{\theta}, D, \gamma$ and $s$ such that $(\bar{p}, \bar{f}, \bar{\theta}, \mathcal{A}, D, \gamma, s)$ is feasible for 15 .

Informally, a pair $(\bar{f}, \mathcal{A})$ is compatible if they give rise to a feasible solution to the safety-constrained problem 15 .

Our implementation relies on two optimization problems that are repeatedly solved in Procedure $\mathrm{V} .3$ given below. We describe these two problems next. Let $0<\tau<1$ be fixed. The first optimization problem is denoted by $\operatorname{Reroute}(\hat{\mathcal{A}}, \tau)$, and uses as inputs a compatible pair $(\hat{f}, \hat{\mathcal{A}})$ and vector $\hat{s}^{2}=\mathbf{V}(\mathcal{A})$ of line flow variances arising from $\hat{\mathcal{A}}$.

$$
\begin{aligned}
& \min _{\bar{p}, \bar{f}, \bar{\theta}} \sum_{i \in \mathcal{G}} c_{i 0}\left(\bar{p}_{i}^{2}+\hat{\mathcal{A}}_{i}^{T} \Omega \hat{\mathcal{A}}_{i}\right)+c_{i 1} \bar{p}_{i}+c_{i 2} \\
& \text { s.t. } \quad B \bar{\theta}=\bar{p}+\mu-d \\
& b_{i j}\left|\bar{f}_{i j}\right|+\nu_{i j} \hat{s}_{i j} \leq(1-\tau) f_{i j}^{\max } \quad \forall i j \in \mathcal{E}, \\
& \bar{f}_{i j}=b_{i j}\left(\bar{\theta}_{i}-\bar{\theta}_{j}\right) \quad \forall i j \in \mathcal{E}, \\
& \forall i \in \mathcal{G}: \\
& p_{i}^{m i n}+\nu_{i} \sqrt{\hat{\mathcal{A}}_{i}^{T} \Omega \hat{\mathcal{A}}_{i}} \leq \bar{p}_{i} \leq p_{i}^{\max }-\nu_{i} \sqrt{\hat{\mathcal{A}}_{i}^{T} \Omega \hat{\mathcal{A}}_{i}} .
\end{aligned}
$$

Comments: This optimization problem minimizes expected generation cost using the fixed participation factors $\hat{\mathcal{A}}$; it imposes stricter line safety constraints (with line limits reduced by the factor $1-\tau)$. Assuming $\operatorname{Reroute}(\hat{\mathcal{A}}, \tau)$ is feasible, let an optimal solution be $\left(\bar{p}^{*}, \bar{f}^{*}, \bar{\theta}^{*}\right)$. Then, by construction of $\operatorname{Reroute}(\hat{\mathcal{A}}, \tau),\left(\bar{f}^{*}, \hat{\mathcal{A}}\right)$ is compatible for $(15)$, with some slack. A large choice for $\tau$ may of course render $\operatorname{Reroute}(\hat{\mathcal{A}}, \tau)$ infeasible. However we will be choosing small values for $\tau$, which as a corollary implies that the expected generation cost accrued by $\bar{p}^{*}$ will be slightly larger than that entailed by the flow $\hat{f}$.

The second problem takes as input a compatible pair $\left(\bar{f}^{\prime}, \mathcal{A}^{\prime}\right)$. Also, for $i j \in \mathcal{E}$ let $s_{i j}^{\prime}=\sqrt{\mathbf{V}\left(\mathcal{A}^{\prime}\right)_{i j}}$, the standard deviation of flow on $i j$ using participation factors $\mathcal{A}^{\prime}$. Finally, let $\mathbf{T}\left(\bar{f}^{\prime}, \mathcal{A}^{\prime}, \tau\right)$ be the set of lines for which the safety constraint is nearly tight under participation factors $\mathcal{A}^{\prime}$, that is to say:

$$
\mathbf{T}\left(\bar{f}^{\prime}, \mathcal{A}^{\prime}, \tau\right)=\left\{i j \in \mathcal{E}:\left|\bar{f}_{i j}^{\prime}\right|+\nu_{i j} s_{i j}^{\prime} \geq(1-\tau) f_{i j}^{\max }\right\} .
$$

The problem, denoted by $\operatorname{VShift}\left(\overline{\mathbf{f}}^{\prime}, \mathcal{A}^{\prime}, \tau\right)$, is as follows.

$$
\begin{aligned}
& \min _{s, \mathcal{A}} \sum_{i j \in \mathcal{F}\left(f^{\prime}\right)} \Delta_{i j}\left(s_{i j}^{2}\right) \\
& \text { s.t. } \quad \mathcal{A} \in \mathcal{K} \\
& s_{i j}^{2} \geq b_{i j}^{2} \pi_{i j}^{T}(I-\mathcal{A}) \Omega\left(I-\mathcal{A}^{T}\right) \pi_{i j} \quad \forall i j \in \mathcal{E} \\
& \left|\bar{f}_{i j}^{\prime}\right|+\nu_{i j} s_{i j} \leq f_{i j}^{\max } \quad \forall i j \in \mathbf{T}\left(f^{\prime}, \mathcal{A}^{\prime}, \tau\right) .
\end{aligned}
$$

Comments. Since the $\Delta_{i j}$ are increasing, at optimality constraint 23c will be binding. Thus the optimization problem is selecting a participation matrix that minimizes the variance metric. Constraint 23d stipulates that on lines $i j \in \mathbf{T}\left(f^{\prime}, \mathcal{A}^{\prime}, \tau\right)$ the matrix $\mathcal{A}$ is compatible with the input flow $f^{\prime}$. Problem 23) is convex and when the $\Delta_{i j}$ are quadratic it is an SOCP; its relative difficulty depends on the number of constraints 23d which, as we have discussed, is frequently quite small.
We now use the two optimization problems to develop an algorithm.

\section{Procedure V.3. Variance-shifting}

Input: Feasible solution $\left(\overline{\mathbf{p}}^{\mathbf{0}}, \mathrm{f}^{\mathbf{0}}, \mathcal{A}^{\mathbf{0}}\right)$ to safetyconstrained problem [15], variance metric $\Delta$, parameters $0<\tau<1, K>0$. Let $\mathbf{s}_{0}^{2}=\mathbf{V}\left(\mathcal{A}_{0}\right)$.

For $k=1,2, \ldots, K$ perform iteration $\mathbf{k}$ :

1. Solve $\operatorname{Reroute}\left(\mathcal{A}_{\mathbf{k}-1}, \tau\right)$. If infeasible, STOP.

Else, let $\left(\overline{\mathbf{p}}^{\mathbf{k}}, \overline{\mathbf{f}}^{\mathbf{k}}, \bar{\theta}^{\mathbf{k}}\right)$ be the optimal solution.

2. Solve VShift $\left(\overline{\mathbf{f}}^{\mathrm{k}}, \mathcal{A}_{\mathbf{k}-\mathbf{1}}, \tau\right)$, with solution $\left(\hat{\mathbf{s}}_{\mathbf{k}}, \hat{\mathcal{A}}_{\mathbf{k}}\right)$.

3. Choose $0<\lambda \leq 1$ largest, so that $\left(\overline{\mathbf{f}}^{\mathbf{k}},(\mathbf{1}-\lambda) \mathcal{A}_{\mathbf{k}-\mathbf{1}}+\lambda \hat{\mathcal{A}}_{\mathbf{k}}\right)$ is compatible.

4. Set $\mathcal{A}_{\mathbf{k}} \leftarrow(\mathbf{1}-\lambda) \mathcal{A}_{\mathbf{k}-1}+\lambda \hat{\mathcal{A}}_{\mathrm{k}}, \mathbf{s}_{\mathbf{k}}^{2}=\mathbf{V}\left(\mathcal{A}_{\mathbf{k}}\right)$.

5. If $\boldsymbol{\Delta}\left(\overline{\mathrm{f}}^{\mathrm{k}}, \mathrm{s}_{\mathbf{k}}^{2}\right) \geq \boldsymbol{\Delta}\left(\overline{\mathrm{f}}^{\mathrm{k}-1}, \mathrm{~s}_{\mathbf{k}-1}^{2}\right)$. STOP. Reset $\tau \leftarrow \tau / 2$.

A formal analysis of this algorithm, which is motivated by Nesterov's smoothing techniques for non-smooth problems $[22]$ is provided in the Appendix. Intuitively, in Step 1 the algorithm reroutes flow so as so as to create slack capacity in lines, while keeping a constant participation matrix (so that variances remain constant). In Step 2 we compute a new participation matrix which is not required to be compatible with the flow vector computed in Step 1, but should improve on the variance metric because of the typically small number of constraints 23d.

Finally, in Steps 3-4 we take a convex combination of the previous and the new participation matrices so as to obtain compatibility. It is straightforward to prove that for any line $i j$, the quantity $\mathbf{V}\left((1-t) \mathcal{A}_{k-1}+t \hat{\mathcal{A}}_{k}\right)_{i j}$ is a convex quadratic function over $0 \leq t \leq 1$ and so the stepsize computation can be performed exactly (see Appendix).

Typically, $\boldsymbol{\Delta}\left(\overline{\mathbf{f}}^{\mathrm{k}}, \hat{\mathrm{s}}_{\mathrm{k}}^{2}\right)<\boldsymbol{\Delta}\left(\overline{\mathbf{f}}^{\mathrm{k}}, \hat{\mathrm{s}}_{\mathbf{k}-1}^{2}\right)$ (since $\left(\hat{\mathbf{s}}_{\mathbf{k}}, \hat{\mathcal{A}}_{\mathbf{k}}\right)$ solves the problem in Step 2). Thus, in order to obtain a large improvement in variance metric, we want the stepsize $\lambda$ to be large. This is the reason why constraint (23d) is needed in problem VShift $\left(\overline{\mathbf{f}}^{\mathbf{k}}, \mathcal{A}_{\mathbf{k}-\mathbf{1}}, \tau\right)$ : without such a constraint a line $i j$ in $\mathbf{T}\left(\bar{f}^{k}, \mathcal{A}^{k-1}, \tau\right)$ would enforce a short step if $\hat{s}_{k, i j}$ is large. Also, as stated the procedure may halt in Step 1. This need not be the case by relying on the expedient of resetting $\tau$ to a smaller value (e.g., half) and repeating Step 1 until feasibility is attained. A more comprehensive solution would be to perform a combination of Step 1 and Step 2, effectively a first-order gradient step, so as to move away from the current point. We have not implemented this patch as it did not prove necessary for small values of $\tau$ and $K$.

\footnotetext{
${ }^{2}$ With $\Delta$ playing the role of a "potential" function.
} 
In the Appendix we will prove an important result concerning the above procedure under model (I) of the variance metric (Section V-A). Let $\Delta^{*}$ be the minimum variance metric over all compatible pairs, i.e.

$$
\Delta^{*} \doteq \min \{\Delta(\bar{f}, \mathbf{V}(\mathcal{A})):(\bar{f}, \mathcal{A}) \text { compatible }\}
$$

Theorem 5. Under model (I) if Procedure (V.3) stops at Step 5 of iteration $k$ then $\boldsymbol{\Delta}\left(\overline{\mathbf{f}}^{\mathbf{k}-\mathbf{1}}, \mathbf{s}_{\mathbf{k}-1}^{\mathbf{2}}\right)=\Delta^{*}$.

\section{NUMERICAL EXAMPLES}

Here we consider numerical examples based on "case2746wp" available through MATPOWER [23], with 2746 buses, 3514 branches, 520 generators and sum of loads 24873. We have added 22 stochastic injection sites, with sum of mean injections 4611.57 (approx. 18.5\% penetration) and average ratio of standard deviation to mean of 0.3 . Our experiment proceeded as follows.

Step 1. First, solve the safety-constrained problem (15) with all safety parameters set to 3 (three standard deviations). All generators are available for balancing. This process required 29 iterations of the cutting-plane algorithm in [3] or [9] and consumed approximately one CPU minute in a standard workstation, using CPLEX $12.6[24]$ as the underlying SOCP solver.

Step 2. We then applied the Procedure V.3 with $\tau=0.1$ and $K=2$. The set of buses used to balance stochastic deviations, $\mathcal{R}$, was the subset of generating buses that was selected in Step 1, of cardinality 11. We expand on Step 2 next.

We used, for variance metric, a nonconvex example of model (II) in Section $\mathrm{V}-\mathrm{A}$. The variance metric we used was

$$
\sum_{i j \in \mathcal{F}} s_{i j}^{2},
$$

i.e. the sum of flow variances in lines in set $\mathcal{F}$. At any iteration $k$ of procedure V.3, the set $\mathcal{F}$ used to define 25 is the union of two sets:

(a) The 100 lines with largest flow.

(b) Those lines for which the safety constraint is nearly binding, i.e. lines $i j$ for which $\left|\bar{f}_{i j}\right|+\nu_{i j} s_{i j} \geq(1-\tau) f_{i j}^{\max }$ ("nearly-binding" lines).

This problem is quintessentially non-convex and it is of interest to see whether our iterative procedure can indeed reduce the metric. A summary of the run is as follows:

Iteration $\mathbf{k}=\mathbf{1}$, Step 1 . Requires 1.12 seconds, optimal expected generation cost $\approx 1.1 \times 10^{06}$.

Iteration $\mathbf{k}=\mathbf{1}$, Step 2. The output of Step 1 produced a set of nearly-binding lines of cardinality 5 ; so that $|\mathcal{F}|=105$, with variance metric (25) of value $6.3 \times 10^{04}$. The optimization problem 23 had approximately 140000 variables and a similar number of constraints and approximately one million nonzeros. Its solution, using Gurobi 7.02 [25], required 2.32 seconds. The variances $\hat{\mathbf{s}}_{1}^{2}$ yield metric $\approx 2.3 \times 10^{04}$.

Iteration $\mathbf{k}=\mathbf{1}$, Step 3. Here, $\lambda \approx 0.55$.
Iteration $k=1$, Steps 4 and 5 . The variances $s_{1}^{2}$ yield a metric of value $\approx 4.65 \times 10^{04}$.

Summary. One iteration of Procedure V.3 keeps expected generation approximately constant and reduces variance metric by approximately $35 \%$.

Iteration $\mathbf{k}=\mathbf{2}$, Step 1. Similar runtime, expected generation cost remains nearly same.

Iteration $\mathbf{k}=\mathbf{2}$, Step 2. The number of nearly-binding branches is now 24 with $\mathcal{F}=120$. Solution statistics are similar to those for iteration 1 . The variance metric attained by $\hat{s}_{2}^{2}$ is $2.89 \times 10^{04}$.

Iteration $\mathbf{k}=\mathbf{2}$, Step 3. Here, $\lambda \approx 0.29$.

Iteration $k=2$, Steps 4 and 5 . The variances $s_{2}^{2}$ yield a metric of value $4.50 \times 10^{04}$.

Summary. Two iterations of Procedure V.3 again keep expected cost nearly constant, but reduce variance metric by roughly $40 \%$ relative to the original value.

It is also instructive to look at the structure of the power flows. At termination, the largest magnitude expected flow is of approximately of value 817 . The third largest flow has value 632 and attains the largest single standard deviation, of value approximately 91 . In contrast, the $101^{\text {st }}$ largest flow value is approximately 144 and lines at or below that ranking of flow magnitude have much smaller standard deviation of flow; approximately 15 . Thus the procedure shifts variance away from high flow lines but also without creating very high variance, low expected flow lines. Of course, other choices for the variance metric $\Delta$ will produce different tradeoffs.

\section{CONCLUSION}

In this work we have described efficient computational procedures that postprocess security-constrained DC-OPF solutions toward nearly-optimal solutions that attain significantly lower variance metrics. In future work we plan to explore alternative metrics, for example to capture engineering details, through the use of derivative-free optimization [26].

\section{ACKNOWLEDGMENT}

This work was supported by the Advanced Grid Modeling Program of the Office of Electricity at the U.S Department of Energy and the Center for Nonlinear Studies at Los Alamos National Laboratory.

\section{REFERENCES}

[1] L. Roald, F. Oldewurtel, T. Krause, and G. Andersson, "Analytical reformulation of security constrained optimal power flow with probabilistic constraints," in Proceedings of the Grenoble PowerTech, Grenoble, France, 2013.

[2] M. Vrakopoulou, K. Margellos, J. Lygeros, and G. Andersson, "A probabilistic framework for security constrained reserve scheduling of networks with wind power generation," in Energy Conference and Exhibition (ENERGYCON), 2012 IEEE International, sept. 2012, pp. $452-457$.

[3] D. Bienstock, M. Chertkov, and S. Harnett, "Chance-constrained DCOPF," SIAM Review, vol. 56, pp. 461-495, 2014.

[4] M. Lubin, Y. Dvorkin, and S. Backhaus, "A robust approach to chance constrained optimal power flow with renewable generation," vol. 31, no. 5, Sept 2016, pp. 3840-3849. 
[5] M. Vrakopoulou and I. A. Hiskens, "Optimal policy-based control of generation and hvdc lines in power systems under uncertainty," in 2017 IEEE Manchester PowerTech, June 2017, pp. 1-6.

[6] L. Roald and G. Andersson, "Chance-Constrained AC Optimal Power Flow: Reformulations and Efficient Algorithms," 2017.

[7] A. Morrison, "Optimized Risk Limits for Stochastic Optimal Power Flow," Master's thesis, ETH, 2016.

[8] D. Phan and S. Ghosh, "Two-stage stochastic optimization for optimal power flow under renewable generation uncertainty," ACM Trans. Model. Comput. Simul., vol. 24, no. 1, pp. 2:1-2:22, Jan. 2014. [Online]. Available: http://doi.acm.org/10.1145/2553084

[9] L. Roald, S. Misra, T. Krause, and G. Andersson, "Corrective Control to Handle Forecast Uncertainty: A Chance Constrained Optimal Power Flow," IEEE Transactions on Power Systems, vol. 32, no. 2, pp. 1626 1637, March 2017.

[10] H. Zhang and P. Li, "Chance constrained programming for optimal power flow under uncertainty," IEEE Transactions on Power Systems, vol. 26, no. 4, pp. 2417-2424, Nov 2011.

[11] K. Sundar, H. Nagarajan, M. Lubin, L. Roald, S. Misra, R. Bent, and D. Bienstock, "Unit commitment with n-1 security and wind uncertainty," in Power Systems Computation Conference (PSCC), 2016. IEEE, 2016, pp. 1-7.

[12] S. Baghsorkhi and I. Hiskens, "Impact of wind power variability on subtransmission networks," in Power and Energy Society General Meeting. IEEE, 2012

[13] M. Davari, F. Toorani, H. Nafisi, M. Abedi, and G. B. Gharehpetian, "Determination of mean and variance of LMP using probabilistic DCOPF and T-PEM," in 2008 IEEE 2nd International Power and Energy Conference, Dec 2008, pp. 1280-1283.

[14] R. Jabr, "A Conic Optimal Power Flow with Uncertain Prices," in Power Systems Computation Conference (PSCC), 2005, 2005, pp. 1-7.

[15] H. Y. Yamin and S. M. Shahidehpour, "Risk and profit in self-scheduling for gencos," IEEE Transactions on Power Systems, vol. 19, no. 4, pp. 2104-2106, Nov 2004

[16] A. Schellenberg, W. Rosehart, and J. Aguado, "Cumulant based stochastic optimal power flow (s-opf) for variance optimization," in IEEE Power Engineering Society General Meeting, 2005, June 2005, pp. 473-478 Vol. 1.

[17] L. Roald, T. Krause, and G. Andersson, "Integrated balancing and congestion management under forecast uncertainty," in 2016 IEEE International Energy Conference (ENERGYCON), April 2016, pp. 16.

[18] D. Bienstock, G. Munoz, C. Matke, and S. Yang, "Robust linear control of storage in transmission systems, and extensions to robust network control problems," 2017.

[19] G. C. Calafiore and L. El Ghaoui, "On distributionally robust chanceconstrained linear programs," Journal of Optimization Theory and Applications, vol. 130, no. 1, pp. 1-22, 2006.

[20] T. Mülpfordt, T. Faulwasser, L. Roald, and V. Hagenmeyer, "Solving Optimal Power Flow with non-Gaussian Uncertainties via Polynomial Chaos Expansion," in $C D C, 2017 . \quad$ IEEE, 2017.

[21] J. Nocedal and S. J. Wright, Numerical Optimization, 2nd ed. New York: Springer, 2006.

[22] Y. Nesterov, "Smooth minimization of non-smooth functions," Math. Program., vol. 103, no. 1, pp. 127-152, May 2005. [Online]. Available: http://dx.doi.org/10.1007/s10107-004-0552-5

[23] R. D. Zimmerman, C. E. Murillo-Sánchez, and D. Gan, "MATPOWER, A MATLAB Power System Simulation Package," IEEE Trans. Power Sys., vol. 26, no. 1, pp. 12-19, 2011.

[24] IBM, "ILOG CPLEX Optimizer," http://www-01.ibm.com/software/ integration/optimization/cplex-optimizer/

[25] GUROBI, "Gurobi Optimizer," http://www.gurobi.com/

[26] S. L. Digabel, "Algorithm 909: NOMAD: Nonlinear Optimization with the MADS algorithm," ACM Transactions on Mathematical Software, vol. 37 , p. $44: 144: 15,2011$

\section{APPENDIX}

Here we provide the technical analysis underpinning Procedure V.3.
Remark 6. Let $\mathcal{A}, \mathcal{A}^{\prime} \in \mathcal{K}$. Then for any $0 \leq t \leq 1,(1-t)+$ $\mathcal{A}+t \mathcal{A}^{\prime} \in \mathcal{K}$ and for any line $i j, \mathbf{V}\left((1-t) \mathcal{A}+t \mathcal{A}^{\prime}\right)_{i j}$ is a convex quadratic function of $t$.

Proof. The first claim follows since $\mathcal{K}$ is convex and the second using expression 8 .

Lemma 7. Suppose that in iteration $k$ the algorithm reaches Step 2. Let $(\bar{f}, \mathcal{A})$ be an arbitrary compatible pair. Then there exists $0<\gamma \leq 1$ such that for all $0 \leq t \leq \gamma$,

$$
\left((1-t) \bar{f}^{k}+t \bar{f},(1-t) \mathcal{A}_{k-1}+t \mathcal{A}\right)
$$

is a compatible pair.

Proof. Consider any line $i j$. For real $0 \leq t \leq 1$ let $s_{i j}^{2}(t)=$ $\mathbf{V}\left((1-t) \mathcal{A}_{(k-1)}+t \mathcal{A}\right)$. By construction in Step 1,

$$
\left|\bar{f}_{i j}^{k}\right|+\nu_{i j} s_{i j}(0) \leq(1-\tau) f_{i j}^{\max } .
$$

Since $s_{i j}^{2}(t)$ is a quadratic function of $t$ and thus continuous, it follows that there exists $\gamma_{i j}>0$ such that

$$
\left|\bar{f}_{i j}^{k}\right|+\nu_{i j} s_{i j}(t) \leq f_{i j}^{\max }
$$

for all $t \leq \gamma_{i j}$. The quantity $\gamma_{i j}$ can be computed exactly using e.g. (8) to obtain an explicit representation of $s_{i j}^{2}(t)$. The result follows using $\gamma=\min _{i j} \gamma_{i j}$.

Recall that $\Delta^{*}$ is the minimum variance metric over all compatible pairs 24, and define

$$
\left(\bar{f}^{*}, \mathcal{A}^{*}\right) \doteq \operatorname{argmin}\{\Delta(\bar{f}, \mathbf{V}(\mathcal{A})):(\bar{f}, \mathcal{A}) \text { compatible }\},
$$

Lemma 8. Suppose that in iteration $k$ the algorithm reaches Step 2 and that $\Delta^{*}<\Delta\left(\bar{f}^{k}, s_{k-1}^{2}\right)$. Let $\gamma$ be as in Lemma 7 and write

$$
s^{2}(\gamma) \doteq \mathbf{V}\left((1-\gamma) \mathcal{A}_{(k-1)}+\gamma \mathcal{A}^{*}\right)
$$

Then

$$
\Delta\left(\bar{f}^{k}, s^{2}(\gamma)\right)<\Delta\left(\bar{f}^{k}, s_{k-1}^{2}\right) .
$$

Proof. Apply Lemma 7 with $\mathcal{A}=\mathcal{A}^{*}$ to obtain $\gamma$ as in (a). To prove (b) consider any line $i j$. Since for any line $i j$, the function $s_{i j}^{2}(t)$ is a convex quadratic function of $t, s_{i j}^{2}(\gamma) \leq$ $(1-\gamma) s_{i j}^{2}(0)+\gamma s_{i j}^{2}(1)$, and therefore, since $\Delta_{i j}\left(s_{i j}^{2}\right)$ is convex and nondecreasing in $s_{i j}$,

$$
\Delta_{i j}\left(s_{i j}^{2}(\gamma)\right) \leq(1-\gamma) \Delta_{i j}\left(s_{i j}^{2}(0)\right)+\gamma \Delta_{i j}\left(s_{i j}^{2}(1)\right) .
$$

Summing this expression over $\mathcal{F}\left(\bar{f}^{k}\right)$ we obtain

$$
\Delta\left(\bar{f}^{k}, s^{2}(\gamma)\right) \leq(1-\gamma) \Delta\left(\bar{f}^{k}, s_{k-1}^{2}\right)+\gamma \Delta^{*}<\Delta\left(\bar{f}^{k}, s_{k-1}^{2}\right)
$$

where the last inequality holds because $\gamma>0$.

Comment: Lemma (8) shows that under very general conditions the variance-shifting Step 2 of Procedure V.3 does lead to a reduction in variance metric. In fact,

Lemma 9. Under model (I) of the variance metric $\Delta\left(\bar{f}^{k}, s_{k}^{2}\right)<\Delta\left(\bar{f}^{k-1}, s_{k-1}^{2}\right)$ unless $\Delta\left(\bar{f}^{k-1}, s_{k-1}^{2}\right)=\Delta^{*}$.

Proof. Under model (I) we have $\mathcal{F}=\mathcal{E}$ in 20). Hence $\Delta\left(\bar{f}^{k}, s_{k-1}^{2}\right)=\Delta\left(\bar{f}^{k-1}, s_{k-1}^{2}\right)$. The result now follows from Lemma 8 In other words we obtain Theorem 5 . 